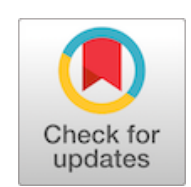

\title{
Cirugía transumbilical asistida versus multipuerto en pacientes pediátricos con diagnóstico de apendicitis aguda
}

\author{
Assisted versus multiport transumbilical surgery for acute appendicitis \\ in pediatric patients
}

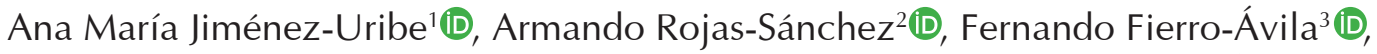 \\ Iván Darío Molina-Ramírez ${ }^{3} \mathbb{D}$, Nicolás Gilberto Mora-Bendeck ${ }^{3} \mathbb{D}$
}

1 Médica, área de hospitalización, Fundación Santa Fe de Bogotá, Bogotá, D.C., Colombia.

2 Médico, especialista en Cirugía pediátrica, jefe de Sección Cirugía Pediátrica, Fundación Santa Fe de Bogotá, Bogotá, D.C., Colombia.

3 Médico, especialista en Cirugía pediátrica, Profesor asociado, Universidad Nacional de Colombia; Fundación Santa Fe de Bogotá, Bogotá, D.C., Colombia.

\section{Resumen}

Introducción. La prevención de las complicaciones en el manejo de la apendicitis aguda sigue siendo un reto para el cirujano pediatra; por lo que es de gran importancia comparar las opciones de manejo quirúrgico, para saber si es posible prevenir dichos resultados, que finalmente llevan a un aumento en el uso de recursos necesarios para tratar a un paciente.

El objetivo de este estudio fue comparar las complicaciones postoperatorias y los costos de la laparoscopia transumbilical asistida y la laparoscopia multipuerto, en pacientes pediátricos en un hospital general de cuarto nivel de la ciudad de Bogotá, D.C., Colombia, entre octubre de 2011 y enero de 2019.

Métodos. Estudio observacional, descriptivo, retrospectivo, de corte transversal, con muestreo no probabilístico a conveniencia, en el que se incluyeron los pacientes entre 0 y 16 años de edad, con historia clínica completa y diagnóstico postquirúrgico de apendicitis aguda, los cuales fueron intervenidos mediante cirugía laparoscópica transumbilical asistida o por multipuerto. Se hizo un análisis descriptivo univariado y bivariado.

Resultados. De los 850 pacientes operados en ese periodo, la técnica quirúrgica más usada fue multipuerto (n=528, 62,1\%) y se presentaron complicaciones en $59(6,94 \%)$ de los pacientes. El diagnóstico postquirúrgico más frecuente fue apendicitis no perforada $(n=762,89,6 \%)$. Al comparar los dos grupos se encontró un valor de $p$ de 0,9685 para la edad, 0,5364 para el diagnóstico postquirúrgico, 0,1127 para las complicaciones postoperatorias y 0,0085 para el costo.

Fecha de recibido: 01/09/2020 - Fecha de aceptación: 31/01/2021 - Fecha de publicación en línea: 19/04/2021 Correspondencia: Ana María Jiménez-Uribe, Carrera 14 B \# 118-58, Apartamento 205, Bogotá, D.C., Colombia. Teléfono: 3166238526. Correo electrónico: anajuribe@hotmail.com

Citar como: Jiménez-Uribe AM, Rojas-Sánchez A, Fierro-Ávila F, Molina-Ramírez IV, Mora-Bendeck NG. Cirugía transumbilical asistida versus multipuerto en pacientes pediátricos con diagnóstico de apendicitis aguda. Rev Colomb Cir. 2021;36:481-6. https://doi.org/10.30944/20117582.757

Este es un artículo de acceso abierto bajo una Licencia Creative Commons - BY-NC-ND https://creativecommons.org/licenses/by-ncnd/4.0/deed.es 
Discusión. El costo de hospitalización y las complicaciones de los pacientes a quienes se les practicó apendicectomía transumbilical asistida es similar a la técnica por multipuerto.

Palabras clave: cirugía pediátrica; apendicitis aguda; apendicectomía; procedimientos quirúrgicos mínimamente invasivos; complicaciones postoperatorias; costos y análisis de costo.

\begin{abstract}
Introduction. The prevention of complications in the management of acute appendicitis remains a challenge for the pediatric surgeon. Therefore, it is of great importance to compare the surgical management options, to know if it is possible to prevent these results, which ultimately lead to an increase in the use of resources necessary to treat a patient. The objective of this study was to compare the postoperative complications and costs of assisted transumbilical laparoscopy and multiport laparoscopy in pediatric patients.
\end{abstract}

Method. Observational, descriptive, retrospective, cross-sectional study with non-probabilistic convenience sampling, where patients between 0 and 16 years old with a complete medical history, with a postsurgical diagnosis of acute appendicitis, who underwent assisted transumbilical surgery or by multiport performed at a fourth level general hospital in Bogotá, Colombia, between October 2011 and January of 2019. A descriptive univariate and bivariate analysis was performed.

Results. Of the 850 patients operated on in this period, the most used surgical technique was multiport ( $\mathrm{n}=528$; $62.1 \%)$ and complications occurred in 59 (6.94\%) of the patients. The most frequent postsurgical diagnosis was non-perforated appendicitis ( $n=762 ; 89.6 \%)$. Comparing the two groups, a $\mathrm{p}$-value of 0.9685 was found for age, 0.5364 for postsurgical diagnosis, 0.1127 for postoperative complications, and 0.0085 for cost.

Discussion. The cost of hospitalization and complications for patients who underwent assisted transumbilical appendectomy is similar to the multiport technique.

Keywords: pediatric surgery; acute appendicitis; appendectomy; minimally invasive surgical procedures; postoperative complications; costs and cost analysis.

\section{Introducción}

La apendicitis aguda es la urgencia quirúrgica más frecuente de la edad pediátrica y su prevalencia alcanza hasta el $11 \%{ }^{1}$. La prevención de complicaciones sigue siendo un reto para el cirujano pediatra; por lo que es de gran importancia la comparación de las opciones de manejo quirúrgico, para evitar resultados adversos, que finalmente llevan a un aumento en el uso de los recursos necesarios para tratar a un paciente.

Dentro del manejo quirúrgico existen dos técnicas mínimamente invasivas ampliamente usadas, la transumbilical asistida (LTA), en la que por medio de un único puerto se accede a la cavidad abdominal, en donde una vez identificado el apén- dice cecal, este se libera y exterioriza, entonces se realiza ligadura de la base por fuera de la cavidad abdominal y sección del apéndice cecal en forma manual; posterior a esto se practica sutura de fascias y piel en la región umbilical ${ }^{2}$ (figura 1).

Por otra parte, existe la laparoscopia convencional, también denominada multipuerto (LMP), en la cual se hace uso de la triangulación para realizar la apendicectomía intraabdominal, con posterior extracción por el puerto umbilical. En esta cirugía es necesario realizar 2 suturas intradérmica adicionales en el tercio inferior del abdomen donde se ubicaron los dos puertos ${ }^{3}$ (figura 2).

La frecuencia de las complicaciones postoperatorias de la apendicectomía varía entre un 


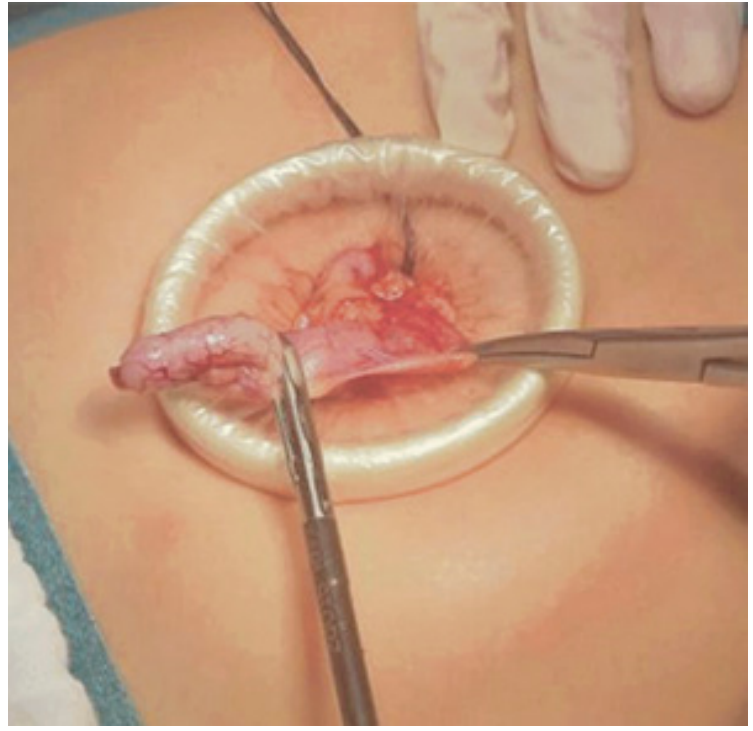

Figura 1. Ligadura de base apendicular en laparoscopia transumbilical asistida.

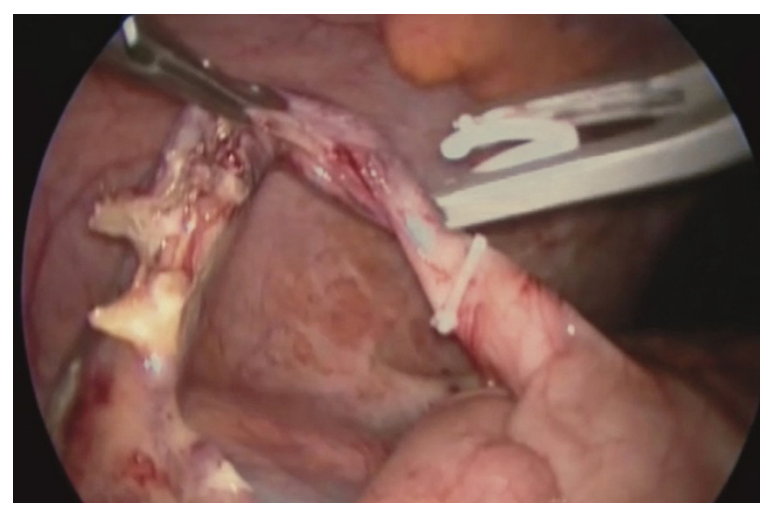

Figura 2. Ligadura de base apendicular en laparoscopia por multipuerto.

10 a $20 \%$. La más común es la infección del sitio operatorio (ISO), que se clasifica, según la profundidad, en incisional superficial, incisional profunda y órgano espacio ${ }^{3,4}$. Usualmente la presencia de complicaciones conlleva a aumentar la estancia hospitalaria de un paciente y los costos de la atención.

$\mathrm{Al} \mathrm{revisar} \mathrm{la} \mathrm{literatura} \mathrm{sobre} \mathrm{estas} \mathrm{dos} \mathrm{técnicas}$ quirúrgicas, se encuentra que hay menor costo y complicaciones con LTA ${ }^{5,6}$, por lo que el objetivo de nuestro estudio fue comparar los dos grupos de pacientes pediátricos con diagnostico de apen- dicitis aguda, sometidos a estos procedimientos laparoscópicos, en un hospital de cuarto nivel de la ciudad de Bogotá, D.C., Colombia, para evaluar si hay diferencias estadísticamente significativas.

\section{Métodos}

\section{Tipo de Estudio}

Estudio observacional, descriptivo, retrospectivo, de corte transversal.

\section{Población y Muestra}

Se incluyeron los pacientes menores de 16 años de edad, con diagnóstico postquirúrgico de apendicitis aguda según reporte de patología, los cuales fueron intervenidos con cirugía laparoscópica, transumbilical asistida o multipuerto, entre octubre del 2011 y enero de 2019, y contábamos con la historia clínica del Hospital Universitario completa.

\section{Instrumento y recolección de datos}

Los datos de los pacientes se tomaron del programa institucional ISIS, el costo de la hospitalización fue extraída del departamento de facturación y el diagnóstico postquirúrgico de apendicitis aguda se obtuvo del reporte de patología de la institución. Los intervalos de costos de hospitalización fueron definidos según la media de los pacientes complicados (10.895.918 COP $=2920,03$ USD) versus no complicados (5.587.044 COP $=1497,2$ USD). Se usó la tasa de cambio del 26 de mayo del 2020 para convertir los costos de pesos colombianos a dólares ( 1 USD = 3731,44 COP)

Los registros fueron consignados en una base de datos codificada, que se procesó en el programa estadístico Epidat 4.2, para posterior análisis estadístico. Se tomaron como variable dependiente la técnica quirúrgica, y como variable independientes edad, sexo, diagnóstico postquirúrgico, complicaciones y costos.

\section{Resultados}

Entre octubre del 2011 y enero de 2019 se operaron 850 pacientes. El 55,1 \% (n=468) eran hombres, el paciente más joven tenía 10 meses de edad, 
el rango de edad más común fue entre los 11 y 16 años, con una media de 10,3 y una mediana de 11 (Tabla 1). En 528 (62,1 \%) pacientes se empleó la técnica LMP y en 322 (37,9\%) pacientes la técnica LTA. El diagnóstico postquirúrgico más frecuente fue apendicitis no perforada (NP), en el 89,6\% $(n=762)$.

Se presentaron complicaciones en un 6,9\% $(n=59)$ de los pacientes, siendo la más común "otras infecciones" (2,2\%), donde se encontró una amplia gama, desde severidad leve, hasta choque séptico de diferentes focos. Entre los pacientes a quienes se les practicó LMP, el 6,3\% $(\mathrm{n}=33)$ presentó complicaciones postquirúrgicas, siendo la más frecuente la ISO incisional superficial, mientras que entre los pacientes a quienes se les practicó LTA, el 8,1\% $(n=26)$ presentó complicaciones, siendo la ISO órgano espacio la más frecuente.
En cuanto a la media de costos de hospitalización, la mayoría de los pacientes estuvo en el rango medio de costo, tanto en el grupo manejado por LMP ( $n=416,78,8 \%)$, como en el grupo con LTA $(n=252,78,3 \%)$, mientras que los pacientes con íleo paralítico u obstrucción por bridas presentaron los porcentajes más altos en el rango mayor de costos (> 2920 USD). La hospitalización más costosa fue de 30.780 USD y correspondió a un paciente operado por LTA que presentó ISO órgano espacio junto con neumonía complicada por un empiema tabicado, y requirió 22 días de estancia en unidad de cuidados intensivos.

El costo promedio de la hospitalización, entre los pacientes complicados, fue de 1944 USD en LMP, con diagnóstico postquirúrgico de apendicitis perforada (PF) en un 41,2 \% ( $\mathrm{n}=14)$, mientras fue de 4158 USD en LTA, con un porcentaje de $60,7 \%(n=17)$ de pacientes con PF (Tabla 1$)$.

Tabla 1. Comparación entre cirugía laparoscopia transumbilical asistida y multipuerto

\begin{tabular}{|c|c|c|c|c|c|c|c|}
\hline \multirow[t]{2}{*}{ Variable } & \multicolumn{2}{|c|}{ Total } & \multicolumn{2}{|c|}{ Multipuerto } & \multicolumn{2}{|c|}{$\begin{array}{c}\text { Transumbilical } \\
\text { asistida }\end{array}$} & \multirow[t]{2}{*}{ Valor $p$} \\
\hline & $\mathbf{N}$ & $\%$ & $\mathbf{N}$ & $\%$ & $\mathbf{N}$ & $\%$ & \\
\hline & 850 & & 528 & $62,1 \%$ & 322 & $37,9 \%$ & \\
\hline Sexo & & & & & & & 0,8304 \\
\hline Femenino & 382 & $44,9 \%$ & 233 & $44,1 \%$ & 149 & $46,3 \%$ & \\
\hline Masculino & 468 & $55,1 \%$ & 295 & $55,9 \%$ & 173 & $53,7 \%$ & \\
\hline \multicolumn{8}{|l|}{ Edad } \\
\hline 0-5 años & 88 & $10,3 \%$ & 54 & $10,2 \%$ & 34 & $10,5 \%$ & 0,9685 \\
\hline 6-10 años & 314 & $36,9 \%$ & 194 & $36,7 \%$ & 120 & $37,3 \%$ & \\
\hline $11-16$ años & 448 & $52,7 \%$ & 280 & $53,0 \%$ & 168 & $52,2 \%$ & \\
\hline Diagnóstico postquirúrgico & & & & & & & 0,5364 \\
\hline Apendicitis no perforada & 762 & $89,6 \%$ & 476 & $90,2 \%$ & 286 & $88,8 \%$ & \\
\hline Apendicitis perforada & 88 & $10,4 \%$ & 52 & $9,8 \%$ & 36 & $11,2 \%$ & \\
\hline Complicaciones & & & & & & & 0,1127 \\
\hline Infección incisional superficial & 16 & $1,9 \%$ & 11 & $2,1 \%$ & 5 & $1,5 \%$ & \\
\hline Infección órgano espacio & 17 & $2,0 \%$ & 5 & $0,9 \%$ & 12 & $3,7 \%$ & \\
\hline Íleo paralítico & 8 & $0,9 \%$ & 6 & $1,1 \%$ & 2 & $0,6 \%$ & \\
\hline Obstrucción por bridas & 2 & $0,2 \%$ & 1 & $0,2 \%$ & 1 & $0,3 \%$ & \\
\hline Otras infecciones & 19 & $2,2 \%$ & 11 & $2,1 \%$ & 8 & $2,5 \%$ & \\
\hline Sin complicaciones & 791 & $93,1 \%$ & 495 & $93,7 \%$ & 296 & $91,9 \%$ & \\
\hline Costo & & & & & & & 0,0085 \\
\hline$<1497$ USD & 20 & $2,3 \%$ & 6 & $1,1 \%$ & 14 & $4,3 \%$ & \\
\hline $1497-2920$ & 668 & $78,6 \%$ & 416 & $78,8 \%$ & 252 & $78,3 \%$ & \\
\hline$>2920$ & 162 & $19,1 \%$ & 106 & $20,1 \%$ & 56 & $17,4 \%$ & \\
\hline
\end{tabular}


Al comparar los dos grupos, solo se encontró una diferencia significativa para el costo $(p=0,0085)$.

Por otra parte, al comparar los pacientes que presentaron apendicitis NP versus PF, independientemente de la técnica (Tabla 2), se encontraron valores de p estadísticamente significativos para edad, complicaciones y costo. Por ejemplo, fue más frecuente la apendicitis perforada en los niños más pequeños y los costos fueron mayores.

\section{Discusión}

En la literatura se sugiere que LTA es superior a LMP para el manejo de los pacientes con apendicitis aguda en cuanto a costos, complicaciones y disminución del tiempo operatorio ${ }^{5-7}$. En niños está patología quirúrgica y sus desenlaces postoperatorios son de gran importancia ya que es la urgencia más frecuente ${ }^{8}$, por lo que el estudio de su manejo en este grupo etario es indispensable para el sistema de salud.

Es nuestra institución, el rango de edad más usual fue de 11 a 16 años, que concuerda con el pico que tiene esta enfermedad (12 años) ${ }^{9,10}$ y las complicaciones se presentaron en un 6,9\%, una cifra menor a lo reportado en revisiones previas

${ }^{8}$, y que podría indicar que, independientemente de la técnica quirúrgica, el manejo que se está haciendo es superior al esperado.

La incidencia de perforación fue mayor a la esperada en los niños de 0-5 años, posiblemente debido a que en esta edad es más usual la presentación atípica del cuadro, asociado a la incapacidad del paciente para expresar sus síntomas con precisión 9,11.

En nuestra institución sigue siendo más frecuente practicar las apendicectomías por LMP que por LTA. Aunque las complicaciones no presentan una diferencia entre las dos técnicas quirúrgicas, llama la atención que la incidencia de ISO órgano espacio en LTA fue de 3,7 \%, mientras que en LMP fue de $0,9 \%$, así mismo, la proporción de ISO incisional superficial en LTA fue de 1,5 \% y en LMP fue de $2,1 \%$. Las otras complicaciones, como el íleo paralítico, la obstrucción por bridas y "otras infecciones" tuvieron mayores costos, ya que se requieren más días de hospitalización y recursos para manejarlas, mientras que en general, las ISO suelen resolverse con manejos cortos y, en la mayoría de las veces, ambulatorios ${ }^{8}$.

El costo de ambas cirugías se agrupó en el segundo rango (1497 - 2920 USD), pero al evaluar el tercer intervalo (> 2920 USD), se encuentra una prevalencia de LMP del 20\% $(n=106)$ en contraste con LTA con un 17,4\% $(n=56)$ en el grupo de

Tabla 2. Comparación entre apendicitis perforada y no perforada

\begin{tabular}{|c|c|c|c|c|c|c|c|}
\hline & \multicolumn{2}{|c|}{ Total } & \multicolumn{2}{|c|}{ Perforada } & \multicolumn{2}{|c|}{ No perforada } & \multirow[t]{2}{*}{ Valor $p$} \\
\hline & $\mathbf{N}$ & $\%$ & $\mathbf{N}$ & $\%$ & $\mathbf{N}$ & $\%$ & \\
\hline & 850 & 100 & 88 & $10,4 \%$ & 762 & $89,6 \%$ & \\
\hline Edad & & & & & & & 0,0001 \\
\hline $0-5$ años & 88 & $10,4 \%$ & 20 & $22,7 \%$ & 68 & $8,8 \%$ & \\
\hline 6-10 años & 314 & $36,9 \%$ & 33 & $37,5 \%$ & 281 & $36,5 \%$ & \\
\hline 11-16 años & 448 & $52,7 \%$ & 35 & $39,8 \%$ & 413 & $53,7 \%$ & \\
\hline Complicaciones & & & & & & & 0 \\
\hline Infección incisional superficial & 16 & $1,9 \%$ & 3 & $3,4 \%$ & 13 & $1,7 \%$ & \\
\hline Infección órgano espacio & 17 & $2 \%$ & 14 & $15,9 \%$ & 3 & $0,4 \%$ & \\
\hline Íleo paralítico & 8 & $0,9 \%$ & 6 & $6,8 \%$ & 2 & $0,3 \%$ & \\
\hline Obstrucción por bridas & 2 & $0,2 \%$ & 2 & $2,3 \%$ & 0 & $0,00 \%$ & \\
\hline Otras infecciones & 19 & $2,2 \%$ & 6 & $6,8 \%$ & 13 & $1,7 \%$ & \\
\hline Sin complicaciones & 791 & $93,1 \%$ & 60 & $68,2 \%$ & 731 & $95,9 \%$ & \\
\hline Costo & & & & & & & 0 \\
\hline$<1497$ USD & 20 & $2,4 \%$ & 2 & $2,3 \%$ & 18 & $2,4 \%$ & \\
\hline 1497-2920 USD & 668 & $78,6 \%$ & 25 & $28,4 \%$ & 643 & $84,4 \%$ & \\
\hline > 2920 USD & 162 & $19,1 \%$ & 61 & $69,3 \%$ & 101 & $13,2 \%$ & \\
\hline
\end{tabular}


LTA, que sugiere diferencias entre ambos grupos, con un valor de p estadísticamente significativo. Esto podría estar asociado a la forma en la que se definieron rangos más que a la técnica quirúrgica, ya que la distribución en esta categoría es similar.

El diagnóstico postquirúrgico de perforación sigue siendo un predictor de más complicaciones y mayor uso de recursos durante la hospitalización, ya que, al haber un mayor y más extenso compromiso inflamatorio, la recuperación suele tardar más días, así como la respuesta fisiológica suele tener una evolución más tórpida ${ }^{10}$, esto se evidencia al comprobar que las diferencias entre ambos grupos son estadísticamente significativas en cada una de las variables que se estudiaron.

\section{Conclusión}

En nuestra institución, la hospitalización de los pacientes a quienes se les practicó apendicectomía LTA tiene un costo menor similar a LMP, así mismo, las complicaciones entre estos dos grupos no presentan diferencias.

\section{Consideraciones éticas}

Consentimiento informado: Se siguieron las directrices de la Resolución 008430 de 1993 del Ministerio de salud sobre las normas científicas, técnicas y administrativas para la investigación en salud y se obtuvo consentimiento informado por parte de los acudientes de los pacientes. Este estudio se realizó con la aprobación del comité de ética de la Fundación Santa Fe de Bogotá del 20 de mayo de 2019.

Conflicto de intereses: los autores declaran no tener ningún conflicto de interés.

Financiación: autofinanciado.

Contribución de los autores

- Concepción y diseño del estudio: Ana Jiménez-Uribe, Armando Rojas-Sánchez, Fernando Fierro-Ávila, Iván Molina-Ramírez y Nicolás Mora-Bendeck.

- Adquisición de datos: Ana Jiménez-Uribe y Armando Rojas-Sánchez.

- Análisis e interpretación de datos: Ana Jiménez-Uribe y Fernando Fierro-Ávila.

- Redacción del manuscrito: Ana Jiménez-Uribe y Fernando Fierro-Ávila.

- Revisión crítica: Armando Rojas-Sánchez, Iván MolinaRamírez y Nicolás Mora-Bendeck.

\section{Referencias}

1. Ziegler MM, Azizkhan RG, Von Allmen D, Weber TR. Operative pediatric surgery. 2nd edition. New York: McGraw-Hill Education/Medical; 2014SE - xxv, 1397 p.

2. Gupta DK, Sharma S, Azizkhan RG. Pediatric surgery: diagnosis and management. New Delhi: Jaypee Brothers Medical Publishers ; 2010.

3. Esposito C, Escolino M, Till H, Bertozzi M, Riccipetitoni G, Settimi A, et al. One-trocar versus multiport hybrid laparoscopic appendectomy: What's the best option for children with acute appendicitis? Results of an international multicentric study. Surg Endosc. 2016;30:4917-23.

https://doi.org/10.1007/s00464-016-4832-y

4. Centers for Disease Control and Prevention. Procedure-associated Module: Surgical Site Infection (SSI) Event, January 2018. Disponible en:

https://www.cdc.gov/nhsn/PDFs/pscManual/9pscSSIcurrent.pdf

5. Kulaylat AN, Podany AB, Hollenbeak CS, Santos MC, Rocourt DV. Transumbilical laparoscopic-assisted appendectomy is associated with lower costs compared to multiport laparoscopic appendectomy. J Pediatr Surg. 2014;49:1508-12.

https://doi.org/10.1016/j.jpedsurg.2014.03.016

6. Jin SG, Cho SH, Kim KY, Ahn SK, Hwang JW, Cho JW, et al. Transumbilical single-incision laparoscopic-assisted appendectomy (TULAA) is useful in adults and young adolescents: comparison with multi-port laparoscopic appendectomy. Medicina. 2019;55:248. https://doi.org/10.3390/medicina55060248

7. Serrano M, Giraldo D, Ordóñez JM, Rengifo HA. Apendicectomía por único puerto asistida por laparoscopia versus técnica abierta convencional localizada, en pacientes con apendicitis aguda en el Hospital Universitario Clínica San Rafael. Rev Colomb Cir. 2019;34:245-53. https://doi.org/10.30944/20117582.438

8. Bratton SL, Haberkern CM, Waldhausen JH. Acute appendicitis risks of complications: age and Medicaid insurance. Pediatrics. 2000;106:75-8. https://doi.org/10.1542/peds.106.1.75

9. Almaramhy HH. Acute appendicitis in young children less than 5 years: review article. Ital J Pediatr 2017;43:15. https://doi.org/10.1186/s13052-017-0335-2

10. Gonzálvez-Bertot J, Kassoum-Djiré M, Paco-Luna H, Díaz-Stevvar M. Caracterización de niños tratados con apendicectomía videolaparoscópica por incisión única. MEDISAN. 2014;18:1671-8.

11. Schwartz KL, Gilad E, Sigalet D, Yu W, Wong AL. Neonatal acute appendicitis: a proposed algorithm for timely diagnosis. J Pediatr Surg. 2011;46:2060-4. https://doi.org/10.1016/j.jpedsurg.2011.07.018 